\title{
IL FATICOSO CAMMINO DEL PD E DELLA SINISTRA NEL NUOVO MILLENNIO
}

di Luciano M. Fasano e Paolo Natale 


\begin{abstract}
In this article we take into consideration the difficult path of the Left in the attempt to overcome the ancient paradigm of identity, intrinsically linked to classical communism. We will retrace the electoral history and the consensus for Left and Centre-left parties from the post-war period to the present. We will analyse the transition from "voto di appartenenza" to the current situation of intense electoral volatility. An empirical analysis of the answers provided by the delegates of the Democratic Party from 2007 to 2013 shows how the heirs of the Communist Party have undergone a constant process of fragmentation, both within the camp and within the parties themselves, The work closes with an attempt to understand (and predict) what the future of the difficult path of the Left within the epochal changes of Western society might be.
\end{abstract}

Keywords: Communist Party, Democratic Party, Left 


\section{Introduzione: la sinistra e la caduta del Muro}

Qualsiasi analisi sull'evoluzione della sinistra italiana dagli inizi degli anni Duemila ad oggi non può che prendere le mosse da qualche considerazione relativa alla fine dell'esperienza del Partito Comunista Italiano, cioè al percorso che si è avviato nel novembre del 1989, all'indomani del crollo del Muro di Berlino, con la cosiddetta "svolta della Bolognina". Questo perché la sinistra italiana del terzo millennio rintraccia $i$ suoi presupposti ideologico-culturali, politici e sociali in quella fase, che coincide con l'avvento del post-comunismo, a seguito della nascita del Partito Democratico della Sinistra - che si affaccerà sul nuovo secolo con il nome di Democratici di Sinistra - e del riproporsi del richiamo alla tradizione comunista che, in quegli stessi anni, verrà rappresentato dapprima dal Partito della Rifondazione Comunista e poi anche dal Partito dei Comunisti Italiani. Senza attribuire la necessaria importanza a quanto accadde negli anni della svolta, non si riuscirebbe infatti a comprendere pienamente i problemi che investono la sinistra italiana di oggi, molti dei quali traggono origine proprio dal modo in cui avvenne il superamento del PCI.

Il 24 novembre 1989, quindici giorni dopo la caduta del Muro di Berlino, partecipando a sorpresa a una manifestazione organizzata dal PCI bolognese per celebrare il $45^{\circ}$ anniversario della battaglia partigiana avvenuta nel quartiere della Bolognina, innanzi a una platea di militanti ed ex partigiani, Occhetto esorta in maniera anche un po' criptica a «non continuare su vecchie strade ma inventarne di nuove per unificare le forze di progresso». E a un giornalista che alla fine del suo discorso gli chiede se le sue parole potessero lasciar presagire il cambiamento di nome del PCI egli, tagliando corto, risponde che «lasciano presagire tutto». Prende così il via, a partire da un atto sostanzialmente unilaterale di quello che sarebbe stato l'ultimo segretario del Partito Comunista Italiano, un lungo e travagliato processo di trasformazione che, con i suoi limiti e le sue contraddizioni, ha ancora oggi per protagonista una sinistra italiana incapace di raggiungere un approdo solido e certo sul piano politico e culturale.

La sinistra italiana vive la trasformazione del PCI in PDS come un evento ineluttabile ma troppo repentino. La necessità di aggiustamenti di rotta, soprattutto in rapporto al peso e all'attualità dell'eredità comunista legata all'impianto teorico del marxismo-leninismo, si era già affacciata in altre importanti occasioni: dal processo di destalinizzazione avviatosi nel 1956, all'indomani della presentazione del Rapporto Kruscev sui crimini di Stalin al XX Congresso del PCUS, alla strategia dell'Eurocomunismo, sancita dal Convegno di Berlino insieme ai partiti comunisti spagnolo e 
francese nel 1976; dall'orizzonte del compromesso storico, inaugurato con la pubblicazione di tre articoli di Berlinguer su Rinascita nel 1973, all'indomani del colpo di stato che destituiva in Cile il governo Allende, e culminato con il governo di solidarietà nazionale insieme alla $\mathrm{DC}$; fino allo strappo con Mosca del 1981, quando a seguito dell'invasione della Polonia da parte dell'Armata Rossa Berlinguer dichiarò che la «spinta propulsiva» della Rivoluzione di ottobre si era ormai esaurita ${ }^{1}$.

In diverse occasioni il PCI aveva preso posizioni che implicavano un cambio di prospettiva rispetto all'ortodossia del comunismo internazionale a guida sovietica. Ma si trattava di semplici variazioni sul tema, aggiustamenti relativi ad articolazioni secondarie dei fini, che non mettevano in discussione la conferma della validità dell'ideologia comunista e il permanere come orizzonte di riferimento del fine ultimo della società socialista (Ignazi, 1992). Ciò che viceversa si verifica nel 1989 è un processo del tutto inedito, che comportando un netto distacco dalla propria tradizione ideologico-culturale, genera pesanti conseguenze sotto il profilo dell'identità politica e della cultura organizzativa del partito.

Si tratta, peraltro, di un passaggio che avviene in circostanze del tutto particolari: dopo alcune pesanti sconfitte subite dal partito, in ambito referendario (con il referendum abrogativo della scala mobile nel 1985), amministrativo (con le elezioni comunali e provinciali del 1986) e politico (con le elezioni per il Parlamento del 1987), che già avevano indotto il gruppo dirigente comunista ad interrogarsi sulla necessità del cambiamento; e dopo l'inattesa scossa prodotta dal crollo del Muro di Berlino (1989), che dà il via alla fine dei regimi comunisti dell'Europa dell'Est e alla lenta ma ormai inesorabile agonia dello stato sovietico, facendo definitivamente svanire il mito e la prospettiva storica dell'esistenza di un'alternativa alla società capitalistica industriale del mondo occidentale.

In questo scritto prenderemo innanzitutto in considerazione il faticoso cammino della sinistra nel tentativo di superare l'antico paradigma identitario, intrinsecamente legato al comunismo classico (par.2); viene poi ripercorsa (par.3) la storia elettorale ed i consensi per i partiti della sinistra e

\footnotetext{
1 Sulla storia del Partito Comunista Italiano rinviamo, per i primi anni della vita repubblicana e fino al 1956, al classico Spriano e Martinelli (1967), mentre per analisi che arrivino fino agli inizi degli anni Novanta, cioè alla conclusione della vicenda di quel partito, si veda Flores e Gallerano (1992), Galli (1993), Agosti (2000), Gualtieri (2001), Vittoria (2006). Per un'interpretazione della storia del PCI inserita nel contesto di una più generale evoluzione della società italiana si rinvia a Ginsborg (1996). Nell'ambito della letteratura internazionale ci limitiamo a ricordare, per la ricostruzione della storia dei comunisti e socialisti italiani nel corso del XX secolo, Alexander e Grand (1989).
} 
del centro-sinistra dal dopoguerra ad oggi, concentrandosi in particolare sulla crisi italiana ma anche europea dell'ultimo decennio. Il paragrafo 4 analizza la trasformazione dell'approccio al voto dei cittadini, passando dal cosiddetto "voto di appartenenza" proprio delle tradizionali subculture di riferimento, che fino agli anni Ottanta garantiva una forte fedeltà al proprio partito, all'attuale situazione di intensa volatilità elettorale, in decisa crescita non soltanto nell'elettorato in generale e in quello di sinistra in particolare, ma anche in quello più motivato come i "selettori", coloro cioè che si mobilitano in occasione delle primarie di partito e di coalizione. La presenza di diverse anime, di diverse concezioni della società e di differenti accentuazioni politiche hanno portato gli eredi del Partito Comunista ad un costante processo di frammentazione, interna sia allo schieramento sia ai partiti stessi, come viene chiaramente evidenziato da un'analisi empirica delle risposte fornite dai delegati del Partito Democratico dal 2007 al 2013 (par.5). Il tentativo di comprendere (e di prevedere) quale possa essere il futuro del difficile cammino della sinistra all'interno degli epocali mutamenti della società occidentale, sia economici che sociali, chiude il presente lavoro (par.6).

\section{La fine dell'identità comunista}

Proprio la drammatica ineluttabilità delle scelte, indotta dal repentino succedersi degli eventi dell'autunno del 1989, innesca un processo di cambiamento che all'interno del gruppo dirigente, e ancor di più nel quadro militante attivo e fra gli iscritti del partito, non ha ancora maturato tutte le condizioni necessarie. Il problema dell'identità comunista deflagra in tutta la sua forza e intensità sia nell'élite comunista che nel suo popolo, ma in maniera quasi speculare (Ignazi, 1992).

Se il gruppo dirigente cerca di fronteggiare la crisi del comunismo ricorrendo a una strategia politica che si sforza di mettere in discussione l'ideologia marxista-leninista, anche se talvolta timidamente e non senza contraddizioni, la base conserva invece una visione politica che rispetto ad alcuni tratti specifici, come la condanna del capitalismo e la critica della società in quanto troppo individualista, mantiene una stretta relazione con un'idea di alternativa di sistema che risente chiaramente di quella stessa ideologia (Baccetti, 1997). Si manifesta così una distanza fra leadership e militanti, rispetto alla quale il ri-orientamento ideologico di stampo 
riformista avviato dai gruppi dirigenti non trova corrispondenza in una convinta adesione degli iscritti (Mulé, 2007).

Il paradosso che sperimenta il PDS, primo erede del PCI, così come i DS, soggetto politico nato in stretta continuità con il PDS nel 1998, fra la cogente necessità di dover cambiare e la evidente incapacità di fare $\mathrm{i}$ conti in via definitiva con la propria storia e la propria ideologia, produce una condizione di indeterminatezza. Uno stato di cose che fin dai tempi della svolta della Bolognina accompagna il tortuoso e difficile cambiamento della sinistra italiana (Ariemma, 2000; Petruccioli, 2001). Un'indeterminatezza che si manifesta con forza anche nel quadro militante attivo del partito (Bellucci, Maraffi e Segatti, 2000), quei delegati congressuali che rappresentano il livello intermedio dell'organizzazione partitica e sono legati alla politica da un'attività semiprofessionale. Perché orientamenti, atteggiamenti e valutazioni di quei delegati possono essere chiaramente descritti in termini di permanenza e discontinuità rispetto al passato comunista.

Così ancora agli inizi degli anni Duemila si può affermare che nel $\mathrm{PDS} / \mathrm{DS}$ convivono elementi sia di forte continuità che di forte discontinuità con il PCI (Bellucci, Maraffi e Segatti, 2000). Anche se la continuità ravvisata dagli studi non è più di carattere ideologico, ma investe prevalentemente l'idea di democrazia rappresentativa, le caratteristiche organizzative e la funzione del partito. In tal senso, il quadro attivo del partito continua a concepire il ruolo dei militanti, il rapporto con gli elettori e le caratteristiche della propria organizzazione in stretta continuità con il modello del partito burocratico a integrazione di massa (Baccetti, 1997), cioè a dire con la concezione - in parte stereotipata e idealizzata - di quello che era il PCI. Un bisogno quasi cognitivo di certezza, che dal piano scivoloso di un'ideologia che non c'è più si sposta sul terreno concreto dell'organizzazione partitica, cercando con una visione idealizzata del soggetto politico di sopperire ad un vuoto di identità politico-culturale che permane (Bellucci, Maraffi e Segatti, 2000). Vi è perciò un importante filo rosso, che lega il popolo di sinistra alla propria idea di appartenenza politica e che dalla svolta della Bolognina assume le vesti dapprima dell'interrogativo su "cosa significhi essere comunista" per poi tradursi nel meno impegnativo quesito su cosa significhi "essere di sinistra", lungo il quale si snoda l'incompiuta traversata del deserto che, passando per differenti formule politiche (PCI-PDS-DS-PD), ha condotto la sinistra italiana fino all'attuale stato di crisi.

Le difficoltà a tracciare una nuova identità che hanno contraddistinto la sinistra che si è resa protagonista di una trasformazione rispetto alla sua 
originaria matrice comunista, nella ricerca di un nuovo ruolo politico e di governo, hanno avuto per contraltare la reazione, radicale e movimentista, della sinistra che rivendicava con orgoglio la sua natura anti-sistema, nella ricerca di un rinnovato incontro con la cultura comunista. Il giorno in cui, a conclusione del XX Congresso del Partito Comunista Italiano, nasce il Partito Democratico della Sinistra, una novantina di delegati guidati dallo storico dirigente del PCI Armando Cossutta, che non si riconoscono nel percorso intrapreso dalla maggioranza occhettiana, si riuniscono sotto le insegne del Movimento per la Rifondazione Comunista, per dare vita a una nuova formazione politica.

Un partito che, fin dalle sue origini, si divide dunque in ragione di due opzioni strategiche fondamentali: salvare il comunismo dal tradimento perpetrato dal PDS di Occhetto, per rifare il partito comunista, oppure prendere atto dei tragici errori del passato e cercare di rifondare il comunismo su radici nuove (Bertolino, 2004). Ma al di là delle differenti opzioni strategiche che alimentano il dibattito interno al PRC, ciò che più pesa, all'indomani del congresso di Rimini, è l'incapacità della sinistra italiana di mantenere aperto questo confronto sugli obiettivi di fondo senza frammentarsi in diversi soggetti politici. Una frammentazione che rappresenterà un tratto costitutivo fino ai giorni nostri, rendendo se possibile ancora più acuta la crisi di identità culturale e politica che attraversa da quasi trent'anni il campo progressista italiano.

Le due opzioni strategiche che caratterizzano il PRC fin dalle origini, fanno sì che in questo nuovo soggetto politico non si ritrovino soltanto i duri e puri della tradizione comunista, ma anche una nuova generazione di militanti per i quali l'essere di sinistra non si esaurisce semplicemente nel filo-sovietismo di Cossutta ma abbraccia tematiche postmaterialiste quali l'ambientalismo, il femminismo, i diritti civili, declinandone i contenuti in una rinnovata chiave antagonista, che pur coniugandosi alle istanze dei nuovi movimenti giovanili conserva gli elementi anticapitalistici e antimperialisti (in particolare di critica verso gli Stati Uniti) della sinistra del passato (Dormagen, 1996). Il modello organizzativo, distaccandosi dalla tradizione del centralismo democratico, favorisce un ruolo attivo dei circoli, cui viene riconosciuta un'autonomia senza precedenti per i partiti dell'epoca e che, di conseguenza, finiscono col rappresentare la vera ossatura portante del nuovo partito (Bertolino, 2004).

Ciò contribuisce a delineare un partito frammentato e difficilmente gestibile, in cui una generazione più anziana cresciuta all'interno della tradizionale subcultura comunista si incontra con una generazione più giovane, che ha fatto nel Sessantotto o nel Settantasette le proprie più 
importanti esperienze di socializzazione e militanza politica, con la permanenza di forti residui ideologici della sinistra extraparlamentare di quegli anni, quali operaismo, trotzkismo, terzomondismo e cattocomunismo (Ignazi, 2018). Ma sarà sotto la segreteria di Fausto Bertinotti, che prenderà la guida del partito al II Congresso nazionale, celebrato nel 1994, che il PRC cercherà di tenere insieme uno spazio politico molto ampio, che va da un'opposizione tradizionale, abituata a trattare in Parlamento, costituita dall'ortodossia della sinistra ex PCI alla più dura opposizione sociale che si innesta con l'antagonismo dei movimenti e gruppi di protesta attivi nel mondo giovanile e nelle aree periferiche delle grandi realtà urbane (Cannavò, 2009). Caratteristica della leadership di Bertinotti è il fatto di coniugare la guida di un partito con molti tratti da opposizione anti-sistema con il tentativo di condizionare l'azione dei partiti di centro-sinistra più moderati, contraddistinti da una vocazione di governo, fino ad influenzarne la strategia delle alleanze, così come le scelte politiche e di indirizzo.

Si risentono già qui, e torneranno ancora più incisivi nel nuovo secolo, gli echi di un'argomentazione che, già qualche decennio addietro, era stata ben evidenziata da un acuto scienziato politico anglo-americano, Henry Drucker (1979): l'incapacità della sinistra di parlare all'intero paese, e non soltanto al proprio elettorato di riferimento. Fin dagli anni Settanta, egli aveva infatti individuato ed analizzato le tre anime che, allora come ora, coesistono all'interno del Labour Party, una commistione che può essere facilmente adattata e attribuita anche ai partiti di sinistra nostrani.

La prima anima è quella di stampo "etico", tipicamente marxiana, basata sulla lotta alle disuguaglianze, sull'egualitarismo, sul riscatto delle fasce più deboli delle popolazioni, per i diritti universali, in difesa dei poveri e dei diseredati di ogni tipo di società.

La seconda anima è quella più "socialdemocratico-laburista", maggiormente legata al proprio elettorato di riferimento, il proletariato inizialmente, la classe operaia e lavoratrice poi, nella sua evoluzione storica, con un'azione politica che ne potesse garantire gli spazi e gli accessi sociali, tramite le conquiste di un welfare diffuso.

La terza anima è quella più "democratico-riformista", il cui intento e obiettivo è quello di governare il paese nel suo complesso, attraverso strumenti e modalità che, per la crescita del paese stesso, si basano su parole d'ordine e azioni politiche meno "liberiste", con un mix delle componenti classiche della dicotomia "stato-mercato".

Secondo il politologo inglese, il prevalere di una o l'altra di queste tre anime, all' interno del partito, condiziona l'attività politica e gli obiettivi che si pone il partito stesso nei confronti del governo del paese. Se 
prevalgono la prima e la seconda anima, ci si limiterà a restare all'opposizione, cercando di condizionare in qualche modo le politiche governative. Se prevale la terza, ci si porrà l'obiettivo di come far funzionare il paese nella sua intera complessità, entrando in aperta competizione con le forze di centro o centro-destra.

Nel primo caso, il referente elettorale resterà confinato in un'area minoritaria della società, più o meno corrispondente al lavoro dipendente, dei cui interessi specifici il partito si farà carico; nel secondo caso, il referente elettorale diventerà di fatto l'intero elettorato, in un'ottica di trasformazione del paese nel suo complesso, ed il partito dovrà essere in grado di sviluppare proposte e politiche appetibili alla maggioranza della popolazione.

Seppur sviluppata negli anni Settanta, questa analisi raffigura in maniera quasi perfetta anche i contorni della situazione italiana così come è emersa nei primi anni della seconda repubblica, fino ai nostri giorni. Bertinotti e Rifondazione Comunista (come più tardi lo stesso Bersani con il "suo" Partito Democratico) rappresentano plasticamente le prime due anime della sinistra, e cercano di condizionare con la loro presenza "esterna" il nascente governo Prodi, nelle elezioni politiche del 1996, pur rifiutandosi di entrare nella coalizione dell'Ulivo guidata da Romano Prodi. Saranno poi il conflitto sugli indirizzi di politica economica-sociale ed estera ad aprire le ostilità fra PRC e governo, che sfoceranno nella crisi sul voto per la legge di bilancio del 1998, con la caduta dell'esecutivo e la conseguente scissione fra il PRC e il Partito dei Comunisti Italiani, nato per iniziativa di un gruppo di parlamentari di Rifondazione capeggiati da Cossutta e Diliberto che, rigettando le direttive del partito, avevano votato la fiducia al governo.

La definitiva radicalizzazione di Rifondazione Comunista su posizioni antagoniste coincide poi con le concitate e drammatiche giornate del Global Social Forum durante il vertice dei G8 a Genova nel 2001, quando il sodalizio con la rete dei movimenti no global viene definitivamente sancito (Cannavò, 2009). Così come all'indomani degli attentati dell'11 settembre dello stesso anno si salda un'intesa con il movimento pacifista che sarà foriero delle diverse manifestazioni contro la guerra in Iraq e l'intervento militare in Afghanistan di cui il PRC sarà promotore, alimentando un conflitto molto forte con i DS sulle questioni di politica estera. Il ritorno di Prodi, che prende il posto di Rutelli alla guida del centro-sinistra in vista delle elezioni politiche del 2006, con l'obiettivo di sconfiggere il fronte di centro-destra berlusconiano spinge Rifondazione Comunista alla ricerca di un'intesa con le altre forze politiche del campo 
progressista, che porterà alla nascita della coalizione dell'Unione e, dopo la vittoria elettorale, all'ingresso del partito nel governo, con la nomina di Paolo Ferrero a Ministro della solidarietà sociale. Ma, di nuovo, la convivenza con gli altri partner dell'esecutivo di centro-sinistra è assai tribolata, costellata com'è dal ripetersi di strappi sia in politica estera sia nelle politiche economiche e sociali, fino alla caduta del II governo Prodi, della quale il PRC viene considerato fra gli artefici (Albertazzi, Mc Donnell e Newell, 2011).

La nascita del Partito Democratico, con la fusione di DS e Margherita alla vigilia delle elezioni politiche del 2008, segna la rottura definitiva dei rapporti fra il PRC e l'ala riformista dello schieramento di centro-sinistra, oltre che l'avvio di un tormentato percorso di riunificazione delle anime della sinistra estranee al $\mathrm{PD}$, che porterà alla formazione di una sorta di cartello elettorale, insieme a Verdi, Comunisti Italiani e Sinistra Democratica, sotto le insegne della Sinistra Arcobaleno. Un'operazione che si dimostrerà elettoralmente fallimentare, costringendo il PRC e i suoi alleati a restare fuori dal Parlamento.

La reazione alla crisi prodotta dall'inattesa estromissione dalla rappresentanza parlamentare porterà in breve tempo alla nascita di due nuove formazioni politiche: Sinistra, Ecologia e Libertà e la Federazione della Sinistra. E sarà poi SEL a raccogliere il testimone di ciò che resta della sinistra italiana fuori dal Partito Democratico, alimentando con quest'ultimo un rapporto dialettico su una serie di temi che vanno dal pacifismo all'ambientalismo, dalla tutela dei lavoratori al riconoscimento dei diritti civili. Un rapporto che sotto la leadership del Presidente della Regione Puglia Nichi Vendola approda alla costruzione di un'intesa elettorale, preceduta dalla partecipazione dello stesso Vendola alle primarie Italia Bene Comune per la selezione del candidato del centro-sinistra a Palazzo Chigi in vista delle elezioni politiche del 2013 (Bordandini, 2013).

Nel frattempo, la tornata amministrativa del 2011 aveva visto il successo, nelle più importanti città al voto (Milano, Genova, Cagliari, Perugia), di candidati espressi o vicini a SEL. Grazie a questi passaggi politici e amministrativi, SEL si accreditava come partner fondamentale della nuova alleanza di centro-sinistra, avviandosi verso le elezioni politiche del 2013, visto il declino del centro-destra di Berlusconi, con buone aspettative di successo (che peraltro non avverrà a causa dell'exploit dell'inedita formazione del Movimento 5 Stelle). Diversa era intanto la situazione in cui si trovavano le altre formazioni politiche di ispirazione radicale e comunista: la Federazione della Sinistra, attraversata da insanabili divisioni, chiudeva i battenti già nel 2012, mentre Rifondazione Comunista 
tornava sulla scena politica proprio alla vigilia delle elezioni politiche del 2013, decidendo di sostenere, insieme a PDCI, Italia dei Valori e Federazione dei Verdi, le liste del nuovo movimento Rivoluzione Civile, legate alla figura dell'ex magistrato Antonio Ingroia.

Il "terremoto elettorale" del 2013 produce però conseguenze inattese, lasciando fuori dal Parlamento Rivoluzione Civile e consegnando a SEL un risultato talmente modesto da sancirne l'irrilevanza politica, vuoi per l'assenza dei numeri al Senato necessari per la formazione di una maggioranza di governo con il PD, e vuoi per la scelta del PD - dopo il fallito dialogo con il M5S - di dare vita a una grande coalizione con Berlusconi e il Popolo delle Libertà. Un ultimo tentativo di risalire la china viene compiuto da SEL alle elezioni europee del 2014, quando insieme ad altre formazioni della sinistra fuori dal PD conia la lista L'altra Europa con Tsipras. Ma con la presenza sulla scena politica di Grillo e del Movimento 5 Stelle, lo spazio per un'iniziativa in grado di rispondere da sinistra alla domanda di protesta dei cittadini, anche in una prospettiva euroscettica e anti-sistema, si è ormai assottigliato. E dopo il non entusiasmante risultato delle elezioni europee SEL si frantuma, in parte confluendo nel Partito Democratico e in parte indirizzandosi verso la nuova ennesima formazione della Sinistra Italiana, guidata dal bersaniano Fassina.

La perenne conflittualità che pervade quest'area politica non accenna peraltro a diminuire, anzi tende ad acutizzarsi ancor più con l'elezione di Renzi come segretario del PD ed il suo successivo approdo a Palazzo Chigi alla guida del governo, anche dentro il Partito Democratico, fra lo stesso Renzi e le diverse componenti della sinistra interna, che faticano a riconoscersi nelle scelte politiche e nello stile del nuovo leader. A seguito di un dissidio che via via diviene sempre più forte, nascono così, oltre a Sinistra Italiana, altre formazioni politiche, in cui si ritrovano sia coloro che decidono di abbandonare il PD in disaccordo con il suo segretario sia coloro che ritengono necessario costruire un soggetto politico di sinistra in grado di contrapporsi all'evoluzione moderata del PD e del suo gruppo dirigente.

L'insieme di questi nuovi gruppi politici, dagli ex bersaniani di Articolo 1-Movimento Democratico e Progressista a Possibile, movimento nato per iniziativa di Pippo Civati, dalla stessa Sinistra Italiana agli ultimi epigoni di SEL, dà vita alla lista Liberi e Uguali, con il proposito di recuperare a sinistra il voto degli elettori delusi dal Partito Democratico. Ma il responso delle elezioni politiche del 2018 è tale da riconsegnare alle due sinistre, quella rappresentata dal PD e quella raccolta sotto le insegne di Liberi e Uguali, un riscontro parimenti deludente, rendendo in questo modo evidente e conclamata quella crisi della sinistra italiana che molto 
probabilmente rintraccia le sue origini in una serie di questioni irrisolte, fin dai tempi della svolta della Bolognina.

\section{Il consenso elettorale della sinistra}

La storia elettorale della sinistra (o del centro-sinistra) in Italia non è mai stata particolarmente felice. In un'unica occasione, sommando le forze comuniste e socialiste dell'epoca con il Partito d'Azione di Parri, è stata superata l'asticella simbolica del $40 \%$ dei voti in una consultazione politica, sebbene sui-generis, come la elezione per l'Assemblea Costituente del lontano 1946. É questo tra l'altro il motivo per cui la nostra Costituzione pareva allora così avanzata, stimolata dalla presenza in parlamento di una forte componente progressista, assente poi negli anni a venire.

Da allora, le sorti dei partiti di sinistra non sono mai più state altrettanto rosee (Capecchi, Polacchini e Galli, 1968), a cominciare dal primo tentativo unitario del 1948, quando il Fronte Democratico Popolare (simboleggiato da Garibaldi) non andò oltre il 31\% dei suffragi elettorali, con un arretramento secco di quasi il $10 \%$ dei voti, in poco meno di due anni.

Con la progressiva uscita del PSI dall'area di sinistra, per approdare al campo governativo democristiano, rimase negli anni soltanto il PCI a rappresentare quello spazio elettorale, con risultati sempre inferiori al 30\%, con l'unica eccezione del 1976, quando pareva avvicinarsi uno storico sorpasso nei confronti dello storico rivale, la DC, separata da soltanto 4 punti percentuali. Ma il terrorismo brigatista da una parte e la proposta (e il timore) di un inedito compromesso storico dall'altra ristabilirono di nuovo, nelle successive elezioni anticipate del 1979, un distacco significativo tra le due formazioni.

Da quell'anno in poi, fino alla fine della cosiddetta prima repubblica e alla quasi contemporanea conclusione dell'esperienza sovietica, l'area di sinistra (ormai identificata con il solo PCI, con l'aggiunta saltuaria di qualche formazione dell'estrema sinistra "extra-parlamentare") non riuscì in quasi nessuna occasione a rappresentare più di un terzo degli italiani che si recavano alle urne. Unica eccezione, per il dovuto omaggio postumo ad Enrico Berlinguer, furono le europee del 1984.

$\mathrm{Fu}$ soltanto con l'avvento della seconda repubblica post-tangentopoli (gli anni definiti in seguito "berlusconiani") che, paradossalmente, riprese vigore elettorale l'appeal dei partiti progressisti, con una declinazione ormai più prossima al centro-sinistra. Includendo poco alla volta anche le 
formazioni legate al cattolicesimo di sinistra, e sommando altresì Rifondazione Comunista, quell'area si trovò a rappresentare stabilmente una quota di elettorato compreso tra il $35 \%$ ed il $40 \%$, trovandosi peraltro molto spesso in minoranza nei confronti delle formazioni ad essa antagoniste, legate appunto al partito di Silvio Berlusconi.

L'unità di intenti per arginare la forza e le proposte politiche di Berlusconi ridiedero slancio elettorale a partiti che, nel contempo, faticavano ad indicare al paese un progetto condiviso in cui identificarsi. Ma nel nome della comune lotta al "berlusconismo" le forze politiche progressiste riuscirono a mietere successi almeno elettorali significativi, che permisero la formazione di un governo di larghe intese, comprendenti anche la stessa formazione di Bertinotti, seppur per un periodo limitato nel tempo.

Il nostro cammino di analisi ha inizio qui. Alla vigilia del nuovo millennio, quando i partiti facenti capo all'Ulivo cominciano ad entrare nell'ottica della costruzione di un nuovo soggetto politico, che sappia unire le istanze dell'ex-comunismo con quelle del cattolicesimo e del laicismo progressista. Il Partito Democratico, nato tra tante speranze verso la fine del primo decennio del nuovo secolo e, oggi, in profonda crisi. Così come in decisa crisi appare tutta l'area del socialismo europeo.

Lo sguardo iniziale di questo nostro cammino nella crisi della sinistra è dunque dedicato proprio alla sinistra europea, da anni in cerca di modalità inedite di ri-pensare alla società contemporanea, e certamente penalizzata sempre più dall'elettorato in generale e, soprattutto, dal suo tradizionale elettorato di riferimento, le classi subalterne.

Come ben si evince anche da un rapido sguardo alla Figura 1 e alla Tabella 1, dove vengono sintetizzati i risultati elettorali dei partiti di matrice socialista nelle rispettive elezioni politiche ${ }^{2}$, a partire più o meno dal $2010 \mathrm{si}$ assiste ad un loro calo generalizzato in quasi tutti i principali paese europei, con la sola eccezione del Regno Unito (e in Svezia), dove il Labour conserva comunque una sua buona capacità elettorale, costantemente tra il 30 ed il $40 \%$ dei voti validi.

\footnotetext{
${ }^{2}$ Nello specifico, sono stati considerati: il Labour (UK), il PASOK (Grecia), il PSOE (Spagna), il PSF (Francia), l'SPD (Germania), il SAP (Svezia) e la coalizione di centrosinistra in Italia.
} 


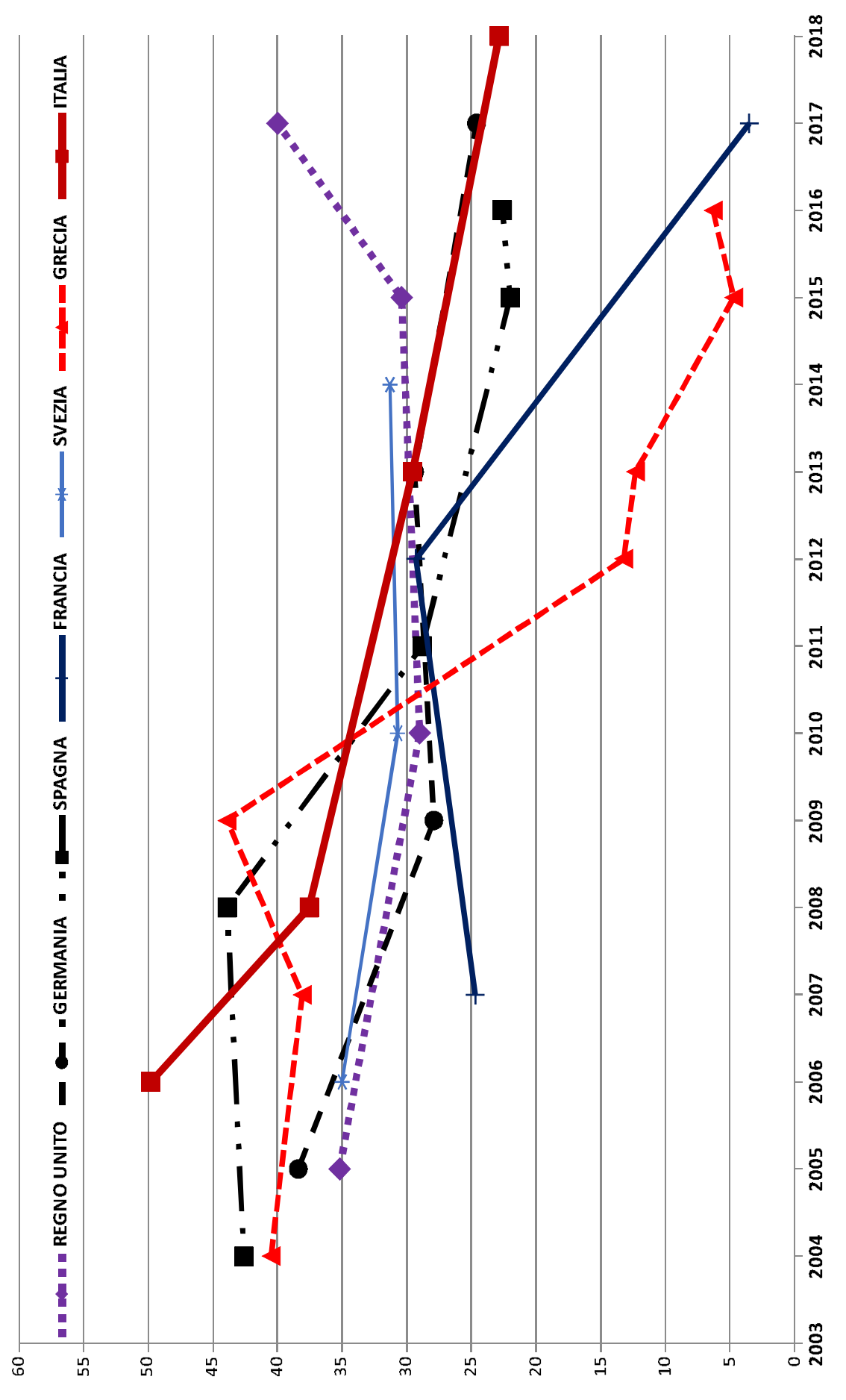

FIG. 1. - Il voto ai partiti del centro-sinistra in Europa, 2004-2018. 


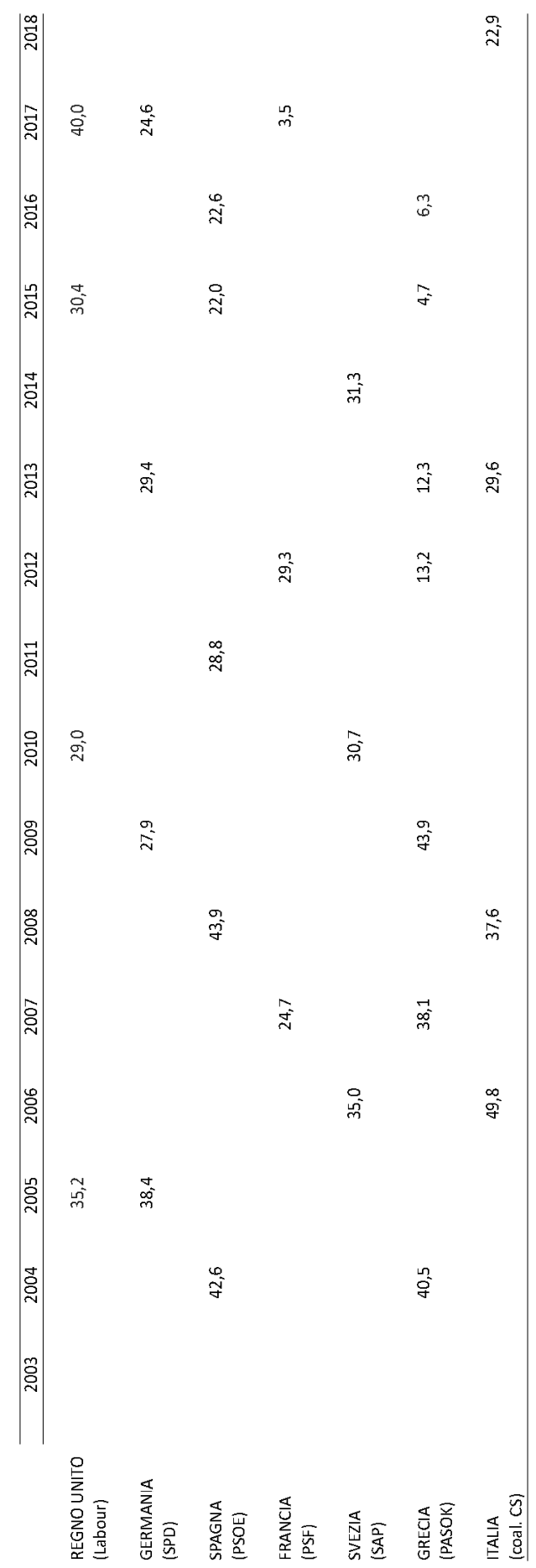

TAB. 1. - Il voto ai partiti del centro-sinistra in Europa, 2004-2018. 
Al contrario, in tutti gli altri paesi, i partiti che fanno riferimento alla tradizione socialista o social-democratica subiscono un crollo dei propri consensi anche di decine di punti percentuali, rispetto al primo decennio del nuovo secolo. Complice la nascita di nuovi raggruppamenti di matrice neopopulista o la rinascita di formazioni politiche apertamente legate alla destra storica, sempre più privilegiati elettoralmente dall'appoggio delle fasce sociali più "deboli", la proposta dei partiti che aderiscono al PSE nel parlamento europeo non incontra più il favore dei cittadini, e la loro performance appare denotata da una costante decrescita dell'appeal elettorale e della fiducia dei cittadini dei rispettivi paesi.

Un "male oscuro" dunque che non tocca soltanto il centro-sinistra italiano, come analizzeremo meglio tra breve, ma tutto il mondo occidentale. Una significativa correlazione tra il voto nelle grandi città, e tra gli strati della popolazione più benestanti, e i partiti di sinistra diviene sempre più evidente in tutte le realtà elettorali, dagli Usa alla Francia, dal Regno Unito alla Spagna, per finire con l'Italia.

La fine degli anni della guerra fredda, chiusa l'esperienza di un mondo spaccato in due blocchi contrapposti (est contro ovest, Nato contro Patto di Varsavia), avrebbe dovuto provocare in tutto il mondo occidentale e nel nostro paese in particolare, dove trovava collocazione il Partito Comunista più forte fra quelli presenti nelle democrazie europee, un ripensamento complessivo della natura della sinistra e della proposta che essa doveva avanzare per il cambiamento socio-politico dei diversi paesi.

Ma, salvo casi isolati, come il ricordato new labour di Tony Blair, così non è stato. A 30 anni dalla caduta del muro di Berlino, la sinistra italiana (come quella europea) paiono dunque ancora oggi alla ricerca del tempo perduto. Lo sguardo è rimasto ancorato ad un mondo che nel frattempo è cambiato, servendosi di categorie culturali e ideologiche del passato, senza una vera rielaborazione della odierna struttura sociale e politica, o del mutato ordine economico-occupazionale legato alla globalizzazione. Un passato che ormai ha perso i suoi ancoraggi storici, in una società in cui, come verrà argomentato nelle prossime pagine, le motivazioni di voto e la rappresentanza politica si nutrono di parole d'ordine così diverse da quelle di un tempo.

La storia elettorale del centro-sinistra in Italia, nel nuovo secolo, non è stata particolarmente differente da quella del passato, negli anni della prima repubblica, quando quell'area (sommando il PCI con le altre piccole formazioni di sinistra, come Democrazia Proletaria) anche allora non otteneva una quantità di voti molto differente da quella di PD e Rifondazione, sempre inferiore al $40 \%$ dei votanti. 
Osserviamo intanto per un attimo il trend dei consensi alla Camera per il centro-sinistra in questo nuovo secolo, dal 2001 ad oggi (Fig.2). Come si può notare, nei primi anni del decennio DS e Margherita, separati (nel 2001) o sotto il simbolo unitario dell'Ulivo (nel 2006), hanno costantemente ottenuto un successo elettorale di poco inferiore ad un terzo dei votanti. L'exploit di Veltroni del 2008, che correva per la prima volta con il neonato Partito Democratico, in realtà non è che sia stato un vero exploit: ha migliorato soltanto di poco, un paio di punti percentuali, il retaggio delle formazioni politiche aggregatisi nel PD e, considerando inoltre il fatto che il resto della sinistra (Rifondazione \& soci) si sia progressivamente ridimensionata, non si può che giungere alla conclusione che il bacino elettorale di quell'area non riuscisse ad andare molto oltre il $35-36 \%$ della popolazione italiana.

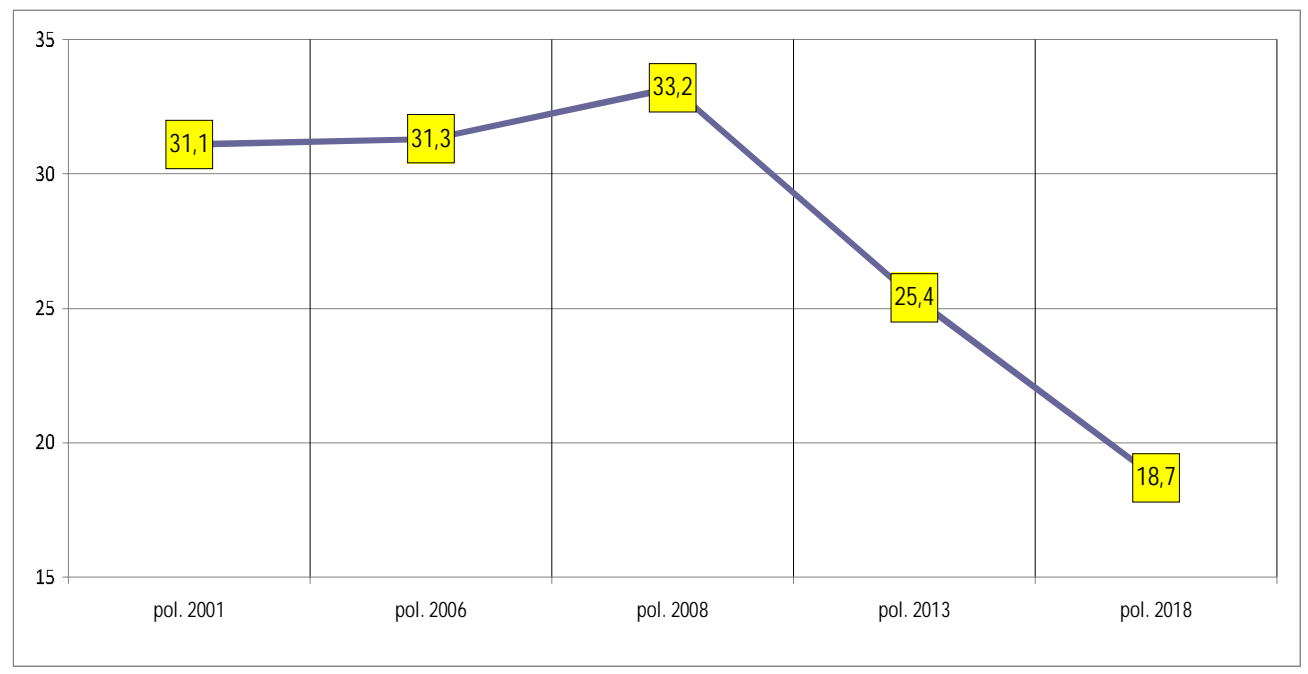

FIG. 2. - Consenso elettorale Camera proporzionale al PD (nel 2001: DS+Margherita; nel 2006: Uniti nell'Ulivo). 
Il Partito Democratico, per certi versi, aveva scommesso sull'allargamento di quella base elettorale, tentando di attirare a sé anche una parte inedita di elettori, che avrebbe potuto guardare alla proposta del $\mathrm{PD}$ con occhi nuovi, diversi dal passato. Il PD, per riuscirci avrebbe dovuto partire dal quel 33\% di Veltroni, incrementando anno dopo anno il suo bacino di consenso.

Ma questo non è accaduto. Anzi, dal 2008 in poi, in tutte le consultazioni legislative il PD è costantemente retrocesso nel favore degli italiani, prima con Bersani ( -8 punti percentuali rispetto a Veltroni) e poi con lo stesso Renzi (un ulteriore $-6,5$ rispetto a Bersani), in una costante e continuativa incapacità di intercettare quei settori sociali cui puntava per accrescere il proprio appeal. Non soltanto infatti il PD non è riuscito a diventare il referente di un nuovo elettorato, ma poco alla volta ha perso sia una parte dei suoi antichi estimatori ex-PCI, con Bersani, che anche dei nuovi, di quelli che avevano sperato in un cambio di prospettiva con Renzi.

E la situazione non cambia di molto se accanto ai partiti fondatori del PD (DS e Margherita) prendiamo in considerazione anche quelli alla sua sinistra (da Rifondazione a SEL, per arrivare a LeU, in tutte le forme di cartello elettorale di cui si sono dotate nel tempo): come è stato sottolineato nel capitolo precedente, in contemporanea con la nascita del Partito Democratico, quell'area raccoglie una quantità di consensi minoritaria, senza riuscire nemmeno in più di una occasione ad entrare nel parlamento.

Né quindi la sinistra riformista né quella più radicale riescono a diventare referenti di un elettorato popolare che poco alla volta vira verso altre mete, prima Berlusconi e Bossi, poi verso il Movimento 5 Stelle e la stessa nuova Lega di Salvini, capaci di catturare il voto di ampi strati della popolazione caratterizzati da un più basso livello socio-economico. L'anima di sinistra pare non esistere più, in nessuna delle sue possibili forme.

\section{Sempre più incerti e infedeli}

Il mondo occidentale nel suo complesso, ed il nostro paese in particolare, ha vissuto come noto per decenni una forte stabilità di comportamento elettorale, quando le motivazioni di voto erano attribuibili per una vasta quota di elettori al cosiddetto "voto di appartenenza», demarcato per questo da un forte livello di fedeltà, una «fedeltà pesante», come è stato spesso definita (cfr. Mannheimer e Natale, 2009, p. 25-26; Natale, 2007, p. 264), frutto del radicamento delle tradizionali sub-culture cattolica, da una parte, e social-comunista, dall'altra. 
Il rapporto del cittadino con il proprio partito era radicato nel suo DNA: il partito era di fatto il punto di riferimento più rilevante nella strutturazione della sua personalità politica, tanto che il sistema normativovaloriale in cui era inserita fin dalla nascita non "permetteva" deviazioni se non occasionali o episodiche. Tramontate dunque, a partire dagli anni Ottanta del secolo scorso, le tradizionali appartenenze sub-culturali, proprie della prima repubblica, si è in parte ricostituita in Italia una inedita spaccatura di tipo ideologico grazie all'avvento di Berlusconi, nella seconda repubblica post-tangentopoli.

La forte contrapposizione tra la visione del mondo del berlusconismo e quella della sinistra ha permesso a quest'ultima di ritrovare un nuovo motivo di "amalgama" tra le sue diverse anime, una sorta di sentimento di vicinanza politico-elettorale quasi in negativo, basata su una conflittualità di resistenza ("ciò che non siamo, ciò che non vogliamo", per dirla alla Montale), ricostruendo una parvenza di appartenenza basata su ciò che è stata definita come «fedeltà leggera» (Natale, 2002; 2007). Il voto riacquistava una nuova forma di stabilità, legata non più all' importanza che il partito rivestiva come rappresentante dei propri interessi, o della propria sub-cultura di riferimento, quanto alla condivisione delle ideologie che le due aree politiche rappresentavano. Destra contro sinistra, stato contro mercato, berlusconismo contro anti-berlusconismo erano, fino alla fine del primo decennio del nuovo secolo, le fratture che determinavano maggiormente la scelta di voto dei cittadini. Ma per la sinistra, il tempo di un ripensamento della propria strategia politica, di una proposta per interpretare lo sviluppo futuro della società occidentale, ed il suo ruolo all'interno di questa società erano di nuovo posticipati, in nome della contingenza elettorale.

Il declino politico di Berlusconi, e la lenta fine dell'epoca berlusconiana, ha lasciato orfano dal 2011 in poi una parte significativa dell'elettorato più vicino alla sinistra, che aveva creduto nella svolta rappresentata dal Partito Democratico, senza però intravedere i confini e i contorni di questa nuova proposta politica.

Così, mentre le analisi dei flussi elettorali del primo decennio del secolo ci mostrano una sostanziale fedeltà di fondo all'interno delle due principali coalizioni, la comparsa di una valida alternativa di voto (rappresentata dal Movimento 5 Stelle e, in parte dal partito di Monti, Scelta Civica) da una parte, ed il progressivo ridimensionamento del "pericolo" rappresentato dalle politiche berlusconiane dall'altra, provoca già nelle elezioni del 2013 un effetto immediato all'interno del precedente elettorato di centro-sinistra. Non riconoscendosi più nel Partito Democratico, nella 
nuova versione di Bersani (così differente da quella di Veltroni), e sentendosi più libero di esplorare nuove vie, una parte considerevole di exelettori PD lo abbandona, andando in diverse direzioni, spesso estranee alla loro tradizione: quasi il $20 \%$ passa al M5S, il $5 \%$ a Scelta Civica e oltre il $10 \%$ decide di astenersi. Mentre un ulteriore $15 \%$ opta per un partito alla sua sinistra (SEL o Rivoluzione Civile di Ingroia), soltanto poco meno del $60 \%$ rimane fedele al proprio voto passato; un livello di fedeltà così basso non si era mai registrato in nessuna altra occasione elettorale precedente per un partito della sinistra italiana, nemmeno considerando come "quasi-fedeli" chi aveva scelto SEL o Ingroia.

Che la fedeltà non rappresenti ormai più un sentimento radicato all'interno del mondo della sinistra, lo testimonia altresì l'atteggiamento manifestato dai cosiddetti "selettori" (i partecipanti alle primarie), che alla classica domanda: «Cosa farà in caso di vittoria del candidato che Lei non ha votato?», sempre più spesso manifestano una crescente alterità alla fedeltà di partito, preferendo invece percorrere strade diverse o, quanto meno, manifestando parecchi dubbi sulle scelte future: astensione, indecisione o perfino un partito diverso dal PD (De Luca e Fasano, 2018). Se gli elettori che confermano la loro fedeltà al partito sono in significativa diminuzione, gli incerti, ovvero coloro per i quali il voto al PD non può darsi per scontato, già nel 2013 (e poi anche nel 2017) rappresentavano quasi un terzo del selettorato complessivo, mentre erano l'11\% solo sei anni prima, nelle primarie 2007 di Veltroni.

É datato dunque 2013, probabilmente, il momento cruciale della storia della sinistra italiana. Da quella elezione in poi il percorso di crisi è delineato. Ed a nulla sono serviti i tentativi, incarnati da Matteo Renzi, di trasformare dall'interno il partito. Dopo il breve idillio tra il nuovo segretario e l'elettorato, culminato con l'ottimo risultato elettorale del 2014 (con oltre il $40 \%$ di suffragi), il declino è ripreso. Renzi era stato capace di convincere una fetta importante di elettori che, con lui, sarebbe iniziato realmente un nuovo corso, un nuovo partito di centro-sinistra che si smarcava dai retaggi del passato, per guardare ad un futuro diverso. Il suo limite, forse, è stato proprio quello di candidarsi ad un modo nuovo di governare, ad una modalità politica inedita per un partito di sinistra, senza averne realmente le capacità "politiche". Il suo fulgore è durato poco, e presto è rientrato nei consueti parametri, inimicandosi inoltre con il suo eccessivo personalismo gran parte di chi aveva per un attimo creduto in lui. Ma probabilmente la vera anomalia è stata proprio quel suo grande successo, che ha fermato per un attimo il declino inevitabile del PD e che poi è ripreso in maniera ineluttabile. Nel 2018, mentre il PD si ferma ad un 
modesto $18,7 \%$, il resto della sinistra non va oltre un ulteriore $4-5 \%$ di consensi: un bacino complessivo pari a meno di un quarto dell'elettorato attivo, il punto più basso della secolare storia della sinistra italiana. Il livello di fedeltà per il PD tocca l'ulteriore minimo storico del 50\%; chi lo abbandona privilegia di nuovo il Movimento 5 Stelle per una quota pari a poco più del 15\% (cfr. Biorcio e Natale, 2018, p.80), l'area dell'astensionismo (17\%) e soltanto il 10\% circa si rivolge a partiti di area (LeU e Bonino).

La sinistra nel suo complesso, sia dunque quella riformista che quella più radicale, esce profondamente trasformata da quest'ultimo appuntamento elettorale. Non soltanto perde molte delle sue storiche roccaforti, nelle cosiddette zone rosse, non soltanto ottiene livelli di consenso così bassi, ma si trova ad avere in dote un elettorato molto diverso da quello tradizionale: il PD viene infatti votato in particolare da anziani e da laureati, ottiene i suoi maggiori successi nelle grandi città e nelle aree più benestanti del paese. Una nuova base di consenso che dovrebbe far riflettere a fondo i dirigenti, reimpostando eventualmente la propria proposta politica, come si dirà nel paragrafo conclusivo.

\section{Tre anime, un partito, più partiti}

La storia degli ultimi trent'anni, dalla svolta della Bolognina in avanti, della sinistra italiana è - come si è visto - costellata da profonde divisioni. La frammentazione politica è l'aspetto che maggiormente si impone all'attenzione di un osservatore: elevato turnover di formazioni politiche, scissioni, aspri conflitti interni ai singoli partiti, profonde e laceranti divisioni fra partiti dello stesso schieramento, difficoltà a costruire una coalizione stabilmente coesa, soprattutto nel momento in cui si tratta di governare.

Diverse sono state le soluzioni tentate, per dare maggior stabilità e organicità all'area di centro-sinistra: prima con l'Unione, poi con l'Ulivo, all' inizio nella veste di una coalizione allargata fra forze politiche di sinistra e di centro-sinistra poi nelle sembianze di una federazione, Uniti nell'Ulivo, che aveva l'ambizione di realizzare un cartello stabile fra i partiti di ispirazione riformista (DS e Margherita) del centro-sinistra, da ultimo il tentativo di dare un baricentro all'intero schieramento attraverso la nascita del Partito Democratico. Ciascuna di queste soluzioni, per ragioni diverse, non è riuscita a sanare le divisioni all'interno di una sinistra in cui si sono via via opposte l'una all'altra anime riformiste e moderate e anime 
movimentiste e radicali, così come formazioni con una spiccata vocazione antagonista e formazioni che ereditavano dal vecchio PCI la propensione alla negoziazione consociativa.

La frammentazione delle forze politiche di sinistra non è una caratteristica esclusiva del nostro paese. Anche in altre realtà, nel corso degli ultimi decenni, si sono riprodotte scissioni e divisioni fra partiti socialisti e socialdemocratici e partiti comunisti (com'è nell'esperienza della sinistra francese e di quella spagnola), oppure fra forze di ispirazione socialista e forze di sinistra radicale (com'è nella più recente esperienza tedesca). Se limitiamo il nostro sguardo ad alcuni paesi membri dell'Unione Europea, oltre alle formazioni ambientaliste, al fianco dei partiti socialisti e socialdemocratici tra i principali partiti di sinistra esterni al campo socialista troviamo in Germania Die Linke, in Francia il PCF e il Partito della Sinistra Radicale, in Spagna Izquierda Unida e Podemos, in Grecia Syriza, in Portogallo il PCP e il Blocco di Sinistra, in Finlandia Alleanza di Sinistra, in Svezia il Partito della Sinistra.

Tale frammentazione si alimenta lungo due tipi di differenze: prima di tutto quelle di ordine tattico, legate ai meccanismi di selezione della rappresentanza interna ed esterna, dai gruppi dirigenti partitici alle candidature nelle liste per l'elezione nelle assemblee legislative, ai diversi livelli (nazionale e locale); quelle poi di ordine strategico, connesse alla formazione degli orientamenti politici e programmatici, all'interno dei partiti, in termini di offerta politica, e fra gli elettori ritenuti di riferimento, esistenti o potenziali, come domanda politica. Tralasciando il primo tipo di cause, che esulano gli scopi del presente contributo, riteniamo opportuno soffermarci sull'analisi del secondo.

Le differenze rispetto agli orientamenti politici e programmatici, come vedremo tra breve, riguardano in primo luogo le questioni economiche e sociali, ovvero l'accettazione o la critica dell'individualismo della società occidentale, dell'economia capitalistica e delle regole del mercato (competizione, opportunità, merito). In secondo luogo, un altro importante aspetto, che influenza indirettamente anche gli orientamenti politici e programmatici, riguarda le modalità di azione considerate prioritarie, che vanno dall'azione antagonista tipica di forze politiche vicine ai movimenti sociali all'azione in sede parlamentare caratteristica dei soggetti politici che provengono dalla tradizione dei partiti socialisti e comunisti.

Non va peraltro dimenticato che la frammentazione politica che contraddistingue la sinistra italiana, al pari della sinistra negli altri paesi europei, forse con l'eccezione della sinistra inglese, non trova riscontro unicamente nella presenza di diversi partiti, ma anche nell'iniziativa politica 
di fazioni e correnti organizzate che, richiamandosi a orientamenti politici e programmatici divergenti, alimentano all'interno dello stesso partito una dialettica talvolta equivalente, per intensità e contrasti, a quella che si ritrova fra differenti partiti dello stesso schieramento. In questo senso, la frammentazione sussiste non soltanto a livello inter-partitico, ma anche a livello intra-partitico. Ne sono un chiaro esempio sia le divisioni nel PRC così come quelle nei DS, nella Margherita e, infine, nello stesso PD.

Peraltro, la persistenza di questi tipi di frammentazione inter e intrapartitica è un dato che contraddistingue l'intero sistema dei partiti, affliggendo anche il centro-destra (Fasano e Pasini, 2014), che nel tempo è parso comunque più tatticamente avveduto, a causa di una particolarità ben nota agli attori politici di quest'ultima area, che hanno infatti cercato di porvi rimedio nel passaggio dalla legge elettorale del "Mattarellum" (dove era prevalente la base coalizionale) a quella del "Porcellum" (dove prevalente era quella partitica): nel centro-sinistra la litigiosità è elevata dal lato dell'offerta ma non nei comportamenti degli elettori, laddove nel centro-destra si verifica(va) una situazione opposta.

Entrando più nello specifico, la frammentazione della sinistra italiana è prevalentemente originata dall'esistenza di diversi orientamenti sulle questioni economiche e sociali. Per avere un'idea più precisa di questo aspetto può essere opportuno guardare a come esso si è finora manifestato all'interno del Partito Democratico, il soggetto politico sul quale disponiamo di una maggiore messe di dati empirici. Grazie a indagini condotte sulla platea dei delegati dell'Assemblea nazionale PD in occasione delle diverse tornate di elezione diretta del segretario è stato possibile ricostruire lo spazio politico di riferimento del quadro militante attivo di quel partito e seguirne l'evoluzione dall'Assemblea costituente del 2007 fino all'Assemblea nazionale eletta nel 2017, a seguito della conferma di Renzi come segretario (Fasano, 2009 e 2010; Fasano e Martocchia Diodati, 2013 e 2017).

Limitandosi alla definizione di uno spazio bidimensionale, dove una prima dimensione etico-valoriale concerne principalmente l'orientamento verso la religione cattolica e su questioni eticamente sensibili (quali le coppie di fatto e l'eutanasia) mentre una seconda dimensione economicosociale riguarda principalmente l'orientamento verso le politiche del lavoro, il ruolo dei sindacati e l'intervento pubblico in economia, la rappresentazione che se ne deriva è quella illustrata nella Figura 3. Il cleavage economico presenta valori negativi in corrispondenza della posizione "pro-labour" e positivi per la posizione "pro-market"; il cleavage etico ha valori negativi in corrispondenza della posizione "pro-choice" e positivi per la posizione "pro-life". In tale spazio politico, trovano 
collocazione le mozioni collegate ai diversi candidati in lizza nelle diverse consultazioni. Candidati a loro volta raccordati attraverso delle linee, così da poter individuare con chiarezza la distanza e l'estensione dello spazio politico coperto dall'insieme delle mozioni di ciascuna tornata elettorale.

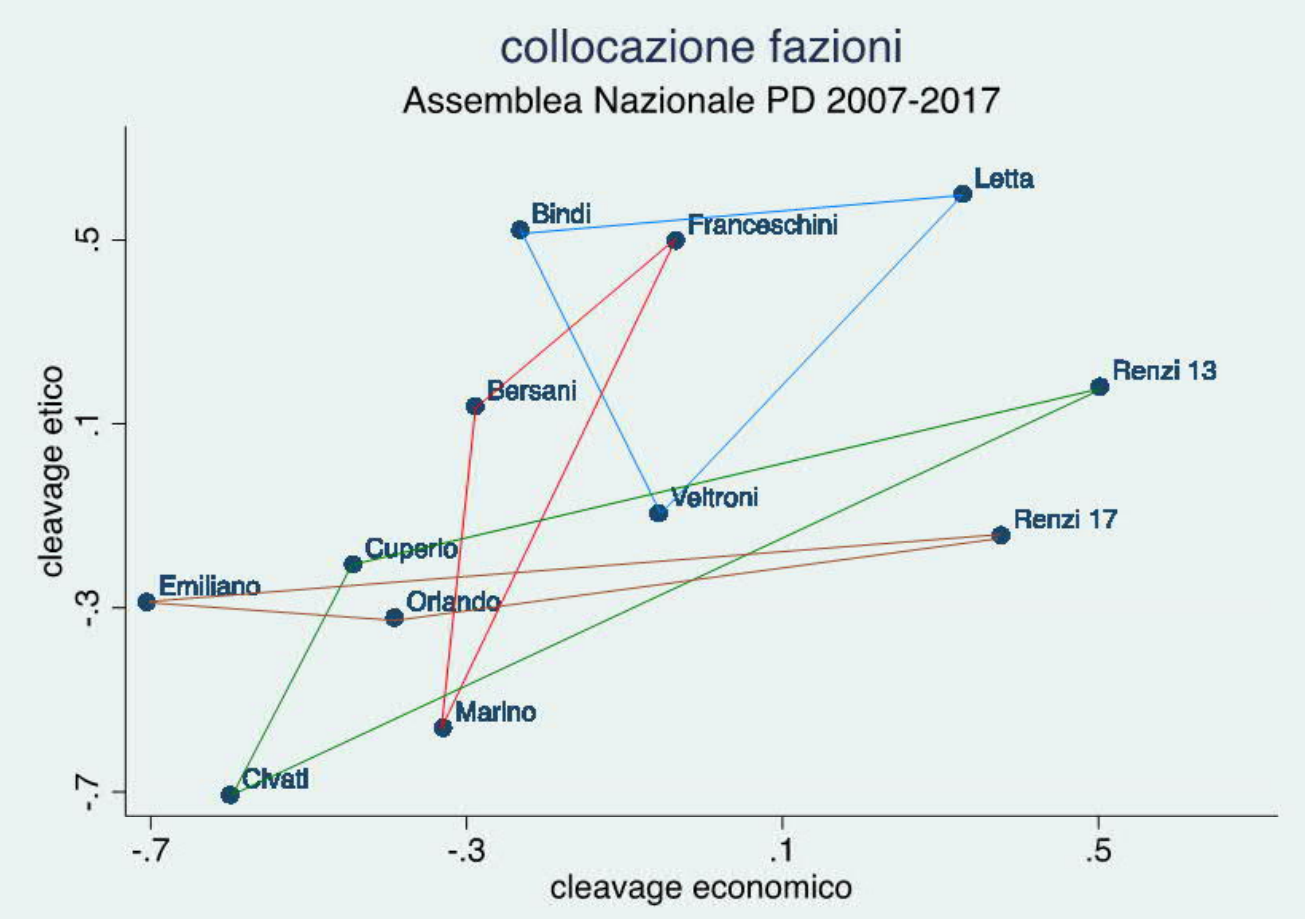

FIG. 3. - Posizionamento spaziale dei delegati del PD dal 2007 al 2013.

Com'è chiaramente visibile, il triangolo delle candidature del 2007 (Veltroni, Bindi e Letta) era collocato in tale spazio in maniera relativamente centrale e le distanze rispetto alle posizioni dei singoli candidati erano piuttosto limitate, sia rispetto al cleavage etico-valoriale sia rispetto a quello economico-sociale ${ }^{3}$. Nel 2009 il triangolo delle candidature

\footnotetext{
${ }^{3}$ La Figura 3 mostra il posizionamento spaziale dei delegati (delle quattro assise nazionali del 2007, 2009, 2013 e 2017), in base alle liste di elezione, nello spazio politico delineato dalle dimensioni definite dall'analisi delle componenti principali. Il cleavage economico presenta valori negativi in corrispondenza della posizione "pro-labour" e positivi per la posizione "pro-market"; il cleavage etico ha valori negativi in corrispondenza della
} 
(Bersani, Franceschini, Marino), pur restando collocato in una posizione relativamente centrale, si contraddistingue per una distanza fra le candidature, in particolare fra Franceschini e Marino, molto più ampia del 2007, soprattutto rispetto al cleavage etico.

È invece con le Assemblee 2013 e 2017, nei due mandati della segreteria renziana, che il triangolo delle candidature si allunga, a dimostrazione di come la distanza fra $i$ candidati diventi sempre più ampia, soprattutto lungo il cleavage economico e a causa della polarizzazione che contraddistingue la candidatura del front-runner (Renzi) rispetto agli altri due.

Con l'eccezione anomala del 2009, a causa della distanza sulla dimensione etica che separa i sostenitori della mozione del laico Marino da quelli della mozione del cattolico Franceschini, è evidente che quella dimensione non rappresenti ormai più un elemento discriminante all'interno del Partito Democratico. É invece sulla dimensione economico-sociale che le differenziazioni assumono un peso di gran lunga maggiore. A dimostrazione di come, esaurito l'entusiasmo e la spinta propulsiva della fase costituente, anche nel PD abbia preso a riprodursi la stessa divisione fra sostenitori di una tradizionale posizione pro-labour (favorevole anche all'intervento pubblico nell'economia) e di una più recente posizione promarket (di accettazione della competizione e del mercato) che si ritrova parimenti nel resto della sinistra italiana.

La nascita del Partito Democratico, quindi, non ha permesso nemmeno alla parte più credibilmente orientata verso aspettative di governo di liberarsi di quel conflitto di fondo riguardante l'accettazione o meno dell'economia capitalistica di mercato, che di fatto continua a rappresentare la principale fronte di opposizione fra le diverse anime della sinistra. E se il potenziale conflitto fra laici e cattolici, con il passare del tempo, ha comunque trovato all'interno del PD una via di ricomposizione, è viceversa il conflitto lungo la dimensione economico-sociale che, con i due mandati della segreteria Renzi, si è accentuato fino a rappresentare la principale linea di opposizione fra maggioranza e minoranze all'interno del partito. Un conflitto che proprio nelle ultime due Assemblee nazionali cambia di segno,

posizione "pro-choice" e positivi per la posizione "pro-life". La ricostruzione dello spazio politico rappresentato nella figura stata possibile grazie alla realizzazione di un'analisi fattoriale, che ha permesso di evidenziare l'esistenza di due fattori latenti in grado di spiegare il $48,3 \%$ della varianza totale. Le variabili prese in considerazione per l'analisi fattoriale sono: il grado d'importanza della religione, il giudizio sull'eutanasia, la severità della legge rispetto all'utilizzo delle droghe leggere, l'insegnamento della religione nella scuola, l'aumento delle tasse a fronte di un aumento dei servizi, l'importanza data al mercato ed alla competizione. 
facendo registrare una netta prevalenza dei sostenitori di una prospettiva pro-market su quelli di una prospettiva pro-labour, ulteriormente accentuata dal sostanziale dimezzamento di questi ultimi, quando in passato $\mathrm{i}$ sostenitori di una prospettiva pro-labour avevano sempre rappresentato la maggioranza dei delegati (vedi Fig. 4).

\section{口pro market pro labour}

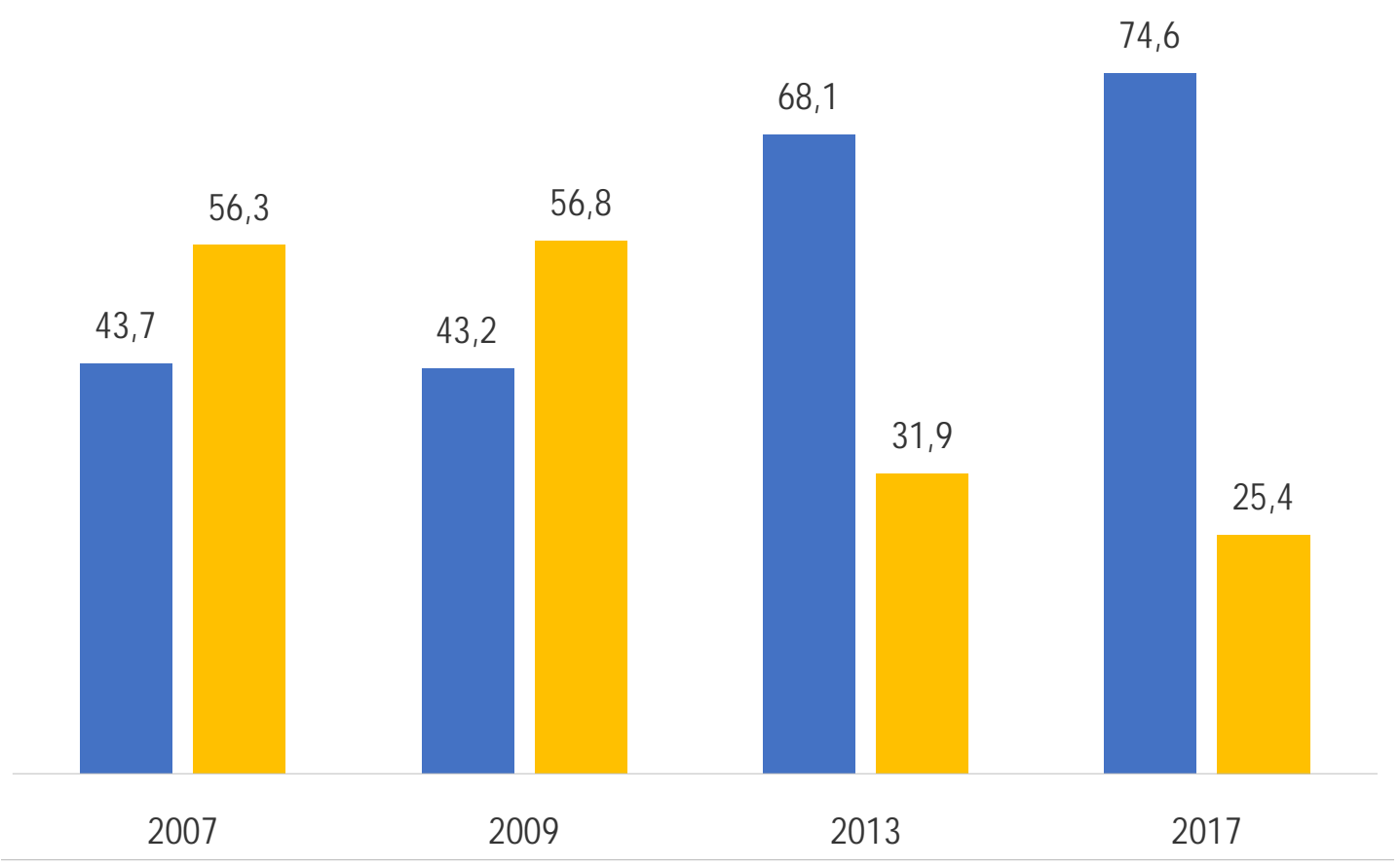

FIG. 4. - Orientamenti sulle politiche economiche e sociali dei delegati alle assemblee del PD. 
Un'altra dimensione rispetto alla quale all' interno del PD, al pari che nel resto della sinistra italiana, si manifestano degli orientamenti critici concerne il giudizio sui processi e gli attori della rappresentanza democratica, rispetto alla quale a un atteggiamento di sostanziale sostegno si contrappone un orientamento protestatario e anti-sistema, che propende per una valutazione negativa sia delle istituzioni democratiche sia dei partiti politici. E ciò che più sorprende, per certi versi, è quanto quest'ultimo atteggiamento sia diffuso all'interno del quadro militante attivo del PD.

Nella platea dei delegati all'Assemblea nazionale, infatti, i detrattori della democrazia rappresentativa oscillano fra il $57,7 \%$ del 2009 , sotto la guida di Bersani, e il 45,2\% del 2017, sotto la guida di Renzi, scendendo al di sotto della soglia del 45\% soltanto all'interno dell'Assemblea costituente del 2007. A dimostrazione di come un giudizio molto critico nei confronti della democrazia e delle sue istituzioni, come portato ideologico di una cultura da sinistra antagonista, che in passato ha trovato ospitalità anche all' interno del PCI, rappresenti un evidente punto di contatto con il mondo del grillismo e del Movimento 5 Stelle, che non interessa soltanto l'elettorato di sinistra ma riguarda da vicino anche i gruppi dirigenti dei suoi partiti di riferimento: una cultura di governo che rimane ancora di là da venire.

\section{Conclusioni: un incerto futuro per la sinistra}

Per la sinistra italiana, al pari di quella degli altri paesi europei, sembra essere arrivato il tempo delle scelte. Il precario stato di salute in cui attualmente versa, com'è plasticamente rappresentato dalla perdita di consensi fatta registrare alle ultime elezioni politiche nelle cosiddette regioni rosse, luogo privilegiato del suo tradizionale insediamento elettorale, richiede un grande e coraggioso sforzo di rinnovamento politico e culturale. Qualora ciò non avvenga la condanna ad una progressiva ma inevitabile emarginazione sembra rappresentare lo scenario più plausibile per il suo futuro.

Dopo aver per lungo tempo rappresentato il punto di riferimento del mondo del lavoro e l'orizzonte di emancipazione delle masse popolari, nel corso degli ultimi 30 anni, dalla caduta del muro di Berlino e dalla svolta della Bolognina, la sinistra ha sostanzialmente cambiato pelle. Ha cercato di fare i conti con l'affermazione storica del capitalismo sul comunismo e si è aperta ai valori della tradizione liberal-democratica, ma secondo modalità alquanto ambivalenti e senza mai esplicitare una scelta decisa e risolutiva. In tal senso, la lenta metamorfosi che ha attraversato nel corso degli ultimi 
trent'anni, non senza resistenze e contraddizioni, non è stata accompagnata dalla costruzione di una nuova identità, così come di una rinnovata cultura politica, che avrebbe dovuto condurre a un riallineamento complessivo fra offerta e domanda politica, fra leader politici, gruppi dirigenti dei partiti e elettori.

La svolta della Bolognina innesca una trasformazione in senso postmaterialista della sinistra italiana, un processo di eterogenesi dei fini in virtù del quale i tradizionali valori della critica anticapitalistica e della centralità del lavoro vengono di fatto progressivamente sostituiti da nuovi valori, declinati in termini di auto-realizzazione e opportunità individuali, così come imposto dai canoni ormai dominanti della società post-industriale. Ma tale svolta fatica a farsi largo anzitutto nel corpo vivo del partito che ne è protagonista, il cui quadro dirigente attivo ancora a distanza di tempo non sembra aver introiettato quelli che avrebbero dovuto diventare i nuovi punti di riferimento di una sinistra democratica, liberale e "orgogliosamente" post-comunista. L'offerta politica della sinistra si polarizza fra posizioni radicali, che vanno dal richiamo alla tradizionale ideologia comunista al riferimento alle nuove forme di antagonismo sociale, e posizioni riformiste, che si sforzano di alimentare una prospettiva di governo in cui il compromesso con l'economia di mercato e la libertà individuale si affermano come nuovi punti di riferimento.

Questo processo di rinnovamento non produce però nuovi consensi nella società. L'elettorato di riferimento è lo stesso di prima, un po' più vecchio, ma comunque fedele, e soprattutto legato a un'idea di sinistra ereditata dalle prime esperienze di socializzazione politica che, se si esclude in assoluto i più anziani, per lo più sono avvenute nel corso della stagione dei movimenti sociali, fra gli anni Sessanta e gli anni Settanta dello scorso secolo. Nel frattempo, la società è cambiata e le nuove generazioni non avvertono più il richiamo della sinistra così come avveniva ai loro predecessori. Perciò anche l'elettorato resta chiuso nei suoi insediamenti tradizionali, sia dal punto di vista territoriale (ma con la recente e paradigmatica disgregazione del consenso anche nelle cosiddette "regioni rosse" delle ultime elezioni politiche) sia dal punto di vista anagrafico.

Un elettorato stabile, che sotto la spinta delle tensioni centrifughe generate dalla dialettica fra sinistra radicale e sinistra riformista si polarizza a sua volta in una parte, sempre più esigua, che risponde positivamente al richiamo degli ideali della sinistra tradizionale e una parte, maggioritaria, che inizia a riconoscersi nella necessità di compromesso imposte dalla responsabilità di governo (perché nel frattempo, con D'Alema, la sinistra ha anche celebrato il suo storico ingresso a Palazzo Chigi). Con ciò, una parte 
degli elettori, che continua ad avvertire come cogente la ricerca di una risposta fondamentalmente ideologica all'interrogativo sulla propria identità, alimenta il dissenso nei confronti della sinistra di governo preferendo, in una logica di pura rappresentanza, i partiti minoritari, che conservano una propensione antagonista da "duri e puri".

Saranno proprio questi elettori, dopo aver sperimentato continue delusioni rispetto alla congruenza fra le loro aspettative identitarie e i comportamenti dei partiti volta per volta votati, a orientare la loro scelta di voto o verso l'exit, l'astensione, ovvero verso forze politiche non di sinistra che si presentano comunque come alternative anti-sistema, a partire soprattutto dal Movimento 5 Stelle. Al tempo stesso, la parte degli elettori che ha deciso, in via più pragmatica, di dare priorità alla competizione per il governo, accantonando - in maniera spesso del tutto acritica - gli interrogativi sulla propria identità politica e culturale, resta fedele al partito che di volta in volta (PDS, DS, PD) incarna la continuità con la storia del maggiore partito della sinistra italiana, il PCI.

Si produce così, nel corso del tempo, una sorta di isolamento dorato del popolo della sinistra, dei suoi gruppi dirigenti e leader politici, che si rispecchia fra offerta e domanda politica, rispettivamente, attraverso la copresenza di frammentazione partitica e polarizzazione elettorale. All'interno di questo quadro, ogni tentativo di semplificazione, dai DS al $\mathrm{PD}$, è inesorabilmente destinato al fallimento, tanto dal generare in ogni occasione, come riflesso conseguente, la nascita di nuovi partiti in contrapposizione al soggetto promotore di una maggiore aggregazione, da PRC ai Comunisti Italiani, da Rivoluzione civile a Sinistra Italiana, fino ad Articolo 1-Movimento Democratici e Progressisti.

Lo stesso ricorso al meccanismo delle primarie, al fine di allargare le maglie della partecipazione mobilitando anche gli elettori nella scelta del leader del PD e del candidato premier della coalizione, non ha avuto tutti gli esiti sperati. Se da un lato, non vi è dubbio che le primarie hanno contribuito ad accrescere il grado di legittimazione di colui che di volta in volta riceveva un'investitura in ragione di una consultazione così ampia e partecipata, dall'altro hanno anche prodotto un più forte accentramento nei processi decisionali all'interno dei partiti e della coalizione, con evidenti conseguenze sul piano della personalizzazione politica, anche positive (Bolgherini e Musella, 2006), che tuttavia non avevano indotto sugli elettori incentivi sufficienti a favorire una semplificazione politica. Nel corso del tempo, infatti, le primarie hanno visto una progressiva smobilitazione degli elettori del PD e del centro-sinistra. Dagli oltre 4 milioni di selettori delle primarie dell'Unione nel 2005, che per diverse ragioni rappresentano un 
caso unico e singolare nel panorama italiano, nelle consultazioni per l'elezione diretta del segretario del PD del 2007 e 2009, così come nel primo turno di quelle per la selezione del candidato premier del centro-sinistra (Italia Bene Comune) del 2012, non si era mai scesi al di sotto della soglia dei tre milioni di elettori.

È invece con le due primarie che hanno visto l'elezione di Renzi segretario, nel 2013 e nel 2017, che si mobilitano rispettivamente 2,8 milioni e 1,8 milioni di elettori, facendo registrare una contrazione del $34,3 \%$ fra queste ultime due tornate. Il decremento della partecipazione fa il paio con la difficoltà manifestata dalle primarie del PD nell'ampliare il coinvolgimento degli elettori di centro-sinistra, come dimostra il fatto che fra una tornata di consultazioni e l'altra le cosiddette matricole, cioè i selettori che partecipano al voto per la prima volta, sono progressivamente diminuiti, passando dal 20,6\% del 2009 al 12,5\% del 2017. A ciò si aggiunga che gli elettori del centro-sinistra mobilitati da queste consultazioni stanno diventando sempre più anziani, evidenziando nel corso dei dieci anni di vita del PD un incremento dell'età media di circa sette anni. È perciò facile concludere che nel medio-lungo periodo le primarie, e quelle del PD più ancora che quelle di coalizione, non siano riuscite a rappresentare quel volano, sia per estendere la partecipazione sia per convogliare gli elettori in direzione del partito maggioritario della coalizione, che era nelle aspettative di coloro che avevano con forza auspicato l'introduzione di questo meccanismo.

Come abbiamo discusso nelle pagine precedenti, tutti questi limiti si sono legati a un dato fondamentale, che resta sullo sfondo dell'evoluzione della sinistra italiana sin dalla fine degli anni Ottanta, e cioè che l'elaborazione del lutto derivante dalla fine del PCI e la ricerca di una nuova identità non si siano mai definitivamente compiute, e la diaspora postcomunista abbia contribuito a rendere ancora più complicata e difficile la costruzione di un orizzonte comune per tutta la sinistra italiana.

Il punto chiave è che il superamento della tradizione comunista, avvenuta repentinamente all'indomani della caduta del muro di Berlino, ha di fatto consumato uno strappo culturale e identitario che non è mai stato colmato. La sinistra italiana, e in particolare il PCI, non ha mai celebrato una Bad Godesberg. Non ha quindi mai fatto in via definitiva i conti con il proprio passato. Perciò l'evoluzione dapprima in una direzione socialdemocratica e poi liberal-socialista non è mai avvenuta attraverso una chiara e consapevole elaborazione politico-culturale. E le leadership che di volta in volta si sono avvicendate sulla scena politica, a cominciare da quelle del partito più grande dello schieramento, non sono mai riuscite nell'intento di 
colmare questo divario. Le vicende che nell'arco di oltre dieci anni hanno contraddistinto la vita del PD, con l'avvicendamento di ben sei segretari alla guida del partito, sette con Zingaretti (con una media di permanenza in ruolo di 1,6 anni ciascuno), ne sono la dimostrazione più evidente. Senza dimenticare come la leadership che nel corso del tempo si è dimostrata più tenace, quella di Renzi che è andato alla riconferma fra il 2013 e il 2017, sia anche quella che maggiormente ha risentito delle irriducibili tensioni esistenti fra le diverse anime della sinistra, che si sono replicate con la massima intensità all'interno del PD fino a produrre diversi abbandoni e scissioni.

Oggi i partiti di centro-sinistra europei, accanto al Partito Democratico nostrano, sono forse gli unici che effettivamente cercano di fare i conti con i millenari mutamenti della società, sia dal punto di vista economico (la globalizzazione e l'insicurezza del lavoro) che sociale (le ondate migratorie e l'ibridazione etnico-culturale). Ma lo fanno, come abbiamo sottolineato, con una scarsa capacità di esplicitare scelte che camminano in direzione per certi versi contraria alla tradizione del mainstream socialista e comunista. Si ritrovano quindi con un elettorato spaccato a metà, gli anziani ex-comunisti ed ex-socialdemocratici da una parte e le aree più di benessere del paese dall'altra; non a caso i consensi maggiori per la sinistra democratica, in molte elezioni nel mondo, giungono proprio, rispetto allo storico cleavage rokkaniano centro-periferia, dalla aree centrali e più ricche (New York, Londra, Parigi o Milano) che anticamente erano quelle più vicine alla destra, mentre le attuali periferie, l'antico bacino elettorale della sinistra storica, dagli anni Novanta in poi appoggiano sempre più chiaramente leader come Trump, Le Pen, Berlusconi e Salvini.

Se le proposte politiche della odierna sinistra paiono a volte più "raffinate" e riflessive, benché esenti da una chiara progettualità per una ipotetica società futura, esse si scontrano quotidianamente con le ondate di problemi e di paure che attanagliano l'immaginario collettivo, il pericolo percepito di una sorta di distruzione dei valori fondanti della civiltà occidentale. Non riescono pertanto ad attecchire se non nell'elettorato più scolarizzato e benestante, che è destinato ovviamente a non diventare mai una forte maggioranza della popolazione elettorale. Una sinistra che si è voluta proporre come valida e capace alternativa nell'assumersi il difficile compito di governare i cambiamenti epocali delle società occidentali, e non solo di denunciarne le criticità stando all'opposizione, rischia di restare confinata ai margini del processo di governance. Per molti anni ancora. 


\section{Nota metodologica}

I dati presentati in Figura 1 ed in Figura 2 sono i risultati ufficiali delle diverse elezioni politiche di ciascuno dei paesi presi in considerazione. Quelli discussi nel paragrafo 4 provengono: i primi, sui flussi di voto, da indagini a livello nazionale su un campione di circa 12 mila intervistati, rappresentativi della popolazione elettorale italiana, effettuati da IPSOS; i secondi, da rilevazioni curate da Candidate \& Leader Selection (C\&LS) sui "selettori" delle cosiddette primarie del PD (circa 4000 casi per ogni "primaria"). Le analisi presentate nel paragrafo 5 (Figure 3 e 4) si basano sulle interviste ai delegati (su un campione pari a circa 400 individui per anno) realizzate nel corso delle Assemblee del Partito Democratico nel 2007, 2009, 2013 e 2017, dove erano presenti i 1000 delegati eletti nelle precedenti primarie. 


\section{Riferimenti bibliografici}

Albertazzi, D., Mc Donnell, D. e Newell, J. (2011), Di lotta e di governo: The Lega Nord and Rifondazione Comunista in Office, in «Party Politics», vol. 17, n. 4, pp. 471-487.

Alexander, J., e Grand, D. (1989), The Italian Left in the Twentieth Century: A history of the socialist and communist parties, Indiana University Press, Bloomington (Ind.)

Accornero, A. (a cura di) (1983), L'identità comunista, Editori Riuniti, Roma.

Agosti, A. (1999), Storia del PCI, Laterza, Roma.

Ariemma, I. (2000), La casa brucia. I Democratici di Sinistra dal PCI ai giorni nostri, Marsilio, Venezia.

Baccetti, C. (1997), Il PDS, il Mulino, Bologna.

Bellucci, P., Maraffi, M. e Segatti, P. (2000), PCI PDS DS. La trasformazione dell'identità politica della sinistra di governo, Donzelli, Roma.

Berlinguer, E. (2014), Per un nuovo grande compromesso storico, Castelvecchi, Roma.

Bertolino, S. (2004), Rifondazione comunista. Storia $e$ organizzazione, il Mulino, Bologna.

Biorcio R. e Natale P. (2018), Il Movimento 5 stelle: dalla protesta al governo, Mimesis, Milano.

Bolgherini, S. e Musella, F. (2006), Le primarie in Italia: ancora e soltanto personalizzazione della politica?, in "Quaderni dell'Osservatorio elettorale», Regione Toscana, vol. 55, n. 1, pp. 219-239.

Bordandini, P. (2013), La spada di Vendola. Una risorsa o un problema per il centro-sinistra?, Donzelli, Roma.

Cannavò, S. (2009), La Rifondazione mancata. 1991-2008, una storia del PRC, Alegre, Roma.

Capecchi, V., Polacchini, V. C. e Galli, G. (1968), Il comportamento elettorale in Italia: una indagine ecologica sulle elezioni in Italia fra il 1946 e il 1963, Istituto di Studi e Ricerche Carlo Cattaneo, il Mulino, Bologna.

Cesarini Sforza, M. e Nassi, E. (1977), L'eurocomunismo, Rizzoli, Milano.

De Luca, R. e Fasano M.L (2018), Il Partito Democratico dei nativi, Epokè, Novi Ligure. 
De Nardis, F. (2009), La Rifondazione comunista. Asimmetrie di potere e strategie politiche di un partito in movimento, Franco Angeli, Milano.

Dormagen, J. Y. (1996), I comunisti: dal PCI alla nascita di Rifondazione comunista: una semiologia politica, Koinè, Roma.

Drucker H. M. (1979), Doctrine and Ethos in the Labour Party, George Allen \& Unwin, Londra.

Fasano, L.M. (2009), L'Assemblea costituente nazionale del PD, in G. Pasquino (a cura di) Il Partito Democratico. Elezione del segretario, organizzazione e potere, Bononia University Press, Bologna.

Fasano, L.M. (2010), L'Assemblea Nazionale del PD, in G. Pasquino e F. Venturino (a cura di) Il Partito Democratico di Bersani. Persone, profilo e prospettive, Bononia University Press, Bologna.

Fasano, L.M. e Martocchia Diodati, N. (2013), L'Assemblea Nazionale del Partito Democratico, in Pasquino, G. e Venturino, F. (a cura di), Il Partito Democratico secondo Matteo, Bononia University Press, Bologna.

Fasano, L.M. e Martocchia Diodati, N. (2017), Dal PD nascenti di Veltroni al PD secondo Matteo di Renzi. Trasformazione di un partito politico e della sua leadership, in "Quaderni di Scienza politica», anno XXIV, n. 1, pp. 7-36.

Fasano, L.M. e Pasini, N. (2014), Tra frammentazione $e$ polarizzazione del sistema politico italiano: interpretazioni e casi empirici, in M. Almagisti, L. Lanzalaco e L. Verzichelli (a cura di), La transizione politica italiana. Da Tangentopoli a oggi, Carocci, Roma.

Flores, M. e Gallerano, N. (1992), Sul PCI, il Mulino, Bologna.

Galli, G. (1993), Storia del PCI, Kaos, Rimini.

Ginsborg, P. (1996), Storia dell'Italia dal dopoguerra a oggi, Einaudi, Torino.

Gualtieri, R. (a cura di) (2001), Il PCI nella storia repubblicana. 1943-1991, Carocci, Roma.

Ignazi, P. (1992), Dal PCI al PDS, il Mulino, Bologna. Bologna.

Ignazi, P. (2018), I partiti in Italia dal 1945 al 2018, il Mulino,

Mannheimer R. e Natale P. (2009), L'Italia dei furbi, il Sole 24 ore, Milano.

Mulé, R. (2007), Dentro i DS, il Mulino, Bologna.

Natale, P. e Fasano, L.M. (2017), L'ultimo partito. Dieci anni di Partito Democratico, Giappichelli, Torino. 
Natale P. (2002), Una fedeltà leggera: $i$ movimenti di voto nella seconda Repubblica, in D'Alimonte R. e Bartolini S. (a cura di), Maggioritario finalmente? La transizione elettorale 1994-2001, il Mulino, Bologna.

Natale P. (2007), Mobilità elettorale e "fedeltà leggera", in Feltrin P., Natale P. e Ricolfi L. (a cura di), Nel segreto dell'urna, UTET, Torino.

Petruccioli, C. (2001), Rendiconto, il Saggiatore, Milano.

Salvati M. (2003), Il Partito Democratico. Alle origini di un'idea politica, il Mulino, Bologna.

Spriano, P. e Martinelli, R. (1967), Storia del Partito comunista italiano: La Resistenza. Togliatti e il partito nuovo, Vol. 5, Einaudi, Torino. 
